\title{
Endovascular Repair of Traumatic Cervical Internal Carotid Artery Injuries: A Safe and Effective Treatment Option
}

\author{
R. Seth, A.M. Obuchowski, and G.H. Zoarski
}

\begin{abstract}
BACKGROUND AND PURPOSE: The appropriate choice of treatment for traumatic extracranial carotid artery injury is still debated. The purpose of this study was to evaluate outcomes of endovascular carotid repair with regard to vessel patency and retreatment rates.

METHODS AND METHODS: We retrospectively reviewed records of patients who underwent endovascular treatment for acute traumatic internal carotid artery dissection with or without pseudoaneurysm formation. The Biffl classification of blunt carotid arterial injuries with an additional modification to stratify grade 2 and 3 injuries into no-flow-limiting (2a/3a) and flow-limiting (2b/3b) was used to classify injuries.
\end{abstract}

RESULTS: Forty-seven patients underwent 50 endovascular interventions. Forty-four were treated with stents alone, 4 required both stent and coil treatments, and 2 were treated with coils alone. Initial treatment resulted in complete restoration of the normal vessel lumen diameter in $25(50 \%)$ treated vessels and good-to-acceptable restoration in $25(50 \%)$ vessels. A single patient had complete stent occlusion. Three patients required stent and/or coil retreatment. There was no mortality or permanent morbidity relating to endovascular carotid artery repair. Twenty-one patients initially treated with medical management ultimately required endovascular treatment. Eighteen $(87.5 \%)$ of these injuries were initially classified as grade $3 a$ and $3(14.3 \%)$ were initially grade 2 a. Injury progression necessitating treatment was identified, on average, within 5 weeks of the initial injury.

CONCLUSIONS: In our series, endovascular therapy was a safe and effective option for restoring luminal caliber and eliminating flow within pseudoaneurysms related to traumatic injuries. Imaging follow-up of all cervicocerebral vascular injuries is especially important within the first 45 days, a critical interval during which most lesions demonstrate healing or progression.

ABBREVIATIONS: ASA = acetylsalicylic acid; $\mathrm{BCVI}=$ blunt cervical vascular injury; $\mathrm{TCAI}$ = traumatic carotid artery injury

T

raumatic vascular injuries of the cervical vertebral and carotid arteries, including vascular dissection and pseudoaneurysm formation, can be the result of blunt or penetrating trauma. These injuries often occur in the setting of rotational, hyperextension, or hyperflexion injury and are frequently related to motor vehicle collisions. Blunt cervical vascular injury is associated with a $12.2 \%$

\footnotetext{
Received March 20, 2012; accepted after revision August 3
}

From the Department of Neuroradiology (R.S.), Northwestern Memorial Hospital, Feinberg School of Medicine of Northwestern University, Chicago, Illinois; Department of Neurointerventional Surgery (G.H.Z.), Christiana Care Health System, Newark, DE; Director of Neurolnterventional Radiology (A.M.O.), JFK Medical Center, Atlantis, FL; Department of Neuroradiology (R.S.), Radiology Associates of South Florida, Baptist Hospital of Miami, Miami, FL; and Assistant Clinical Professor of Radiology (A.M.O.), University of Miami Miller School of Medicine, Miami, FL.

Previously presented as a poster at: 48th Annual Meeting of the American Society of Neuroradiology and Neuroradiology Education and Research Foundation Symposium, May 15-20, 2010; Boston, Massachusetts.

Please address correspondence to Gregg H. Zoarski, MD, Department of Neurointerventional Surgery, Christiana Care Health System, 4755 Ogletown-Stanton Rd, Newark, DE 19178; e-mail: gzoarski@gmail.com

http://dx.doi.org/10.3174/ajnr.A3337 risk of acute cerebral ischemia ${ }^{1}$ and is the cause of stroke in approximately $15 \%$ of patients $16-45$ years of age presenting with acute cerebral ischemia. ${ }^{2}$ Furthermore, patients with accompanying injuries, such as acute cervical spine fracture, basilar skull fracture, major thoracic injury, and mandibular fracture are at risk of vascular injury. ${ }^{1,3}$ The diagnosis of cervical vascular injury in patients with trauma or suspected cervical vascular injury is most commonly confirmed with CTA (Fig 1), MR imaging, duplex sonography, and/or conventional angiography. A recent evaluation of CTA with 16-section CT scanners demonstrated a $97.7 \%$ sensitivity and $100 \%$ specificity compared with the criterion standard of conventional angiography. ${ }^{4}$

Earlier studies suggested that BCVI involving either the carotid or vertebral arteries was present in $0.1 \%$ of patients hospitalized for trauma ${ }^{5}$; however, recent data based on more aggressive screening protocols demonstrate a higher prevalence of BCVI at $1.2 \%-1.6 \%$ of all patients with trauma ${ }^{1,6,7}$ and in $2.7 \%$ of patients with severe multisystem trauma. ${ }^{8}$ Further subdivided, carotid injuries were identified in $0.32 \%$ of all patients with blunt 
trauma, ${ }^{9}$ and vertebral injures were identified in $0.53 \%$ of all patients with blunt trauma. ${ }^{10}$ Penetrating injuries are less commonly seen.

Carotid and vertebral artery injuries may occur "spontaneously" or may be iatrogenic. The pathogenesis of spontaneous injuries may relate to underlying structural or collagen-vascular defects of the arterial wall, including Ehlers-Danlos syndrome, Marfan syndrome, autosomal dominant polycystic kidney disease, osteogenesis imperfecta type I, or other nonspecific abnormalities such as fibromuscular dysplasia and cystic medial necrosis. ${ }^{11}$ Additional factors, such as chiropractic manipulation and unrecognized or remote trauma, may also be significant contributing factors in spontaneous cervical vascular injury. The average annual incidence of these spontaneous injuries has been reported to range between 2.6 and 2.9 per $100,000 .^{12}$

Although risk factors for BCVI have been analyzed to prescribe criteria for screening, ${ }^{13}$ data and recommendations regarding treatment options and outcomes for patients with vascular injuries are more limited. Asymptomatic patients with low-grade injuries are typically treated conservatively with medical management and imaging follow-up. Studies have shown a decrease in neurologic sequelae with the use of antithrombotic or antiplatelet agents in these patients, with no significant difference between the 2 agents. ${ }^{14,15}$ Unfortunately, contraindications to anticoagulants and antiplatelet agents in patients with multisystem injury, including the presence of intracranial hemorrhage, often complicate the treatment paradigm within the trauma population.

Few prospective trials have been performed to evaluate the safety and efficacy of endovascular therapy in patients with acute traumatic carotid artery injury. The use of cervical and intracranial vascular stents and coils in the treatment of TCAI has been described; however, only a few small retrospectives series have been published. The largest of these reviews describes encouraging results for carotid artery stents placed in 29 patients. ${ }^{16}$ In 2005 , however, Cothren et $\mathrm{al}^{17}$ showed a $21 \%$ complication and $45 \%$ occlusion rate following carotid stent placement in patients with grade 3 injuries, while those patients treated with anticoagulation alone demonstrated only a 5\% occlusion rate. Although these data are discouraging, the high occlusion rate in the treatment group may be attributable to the fact that all patients who had complications within the stent arm of the study received pre- and posttreatment with only anticoagulation agents rather than antiplatelet therapy. The retrospective study of 29 patients performed by Kadkhodayan et $\mathrm{al}^{16}$ in 2005 demonstrated a much lower complication rate of $6.9 \%$ with the use of antiplatelet agents pre- and poststenting.

\section{MATERIALS AND METHODS \\ Patient Selection}

Following approval by the institutional review board, we retrospectively reviewed the records and imaging studies of patients with trauma who had undergone endovascular extracranial internal carotid artery repair for acute dissection with or without pseudoaneurysm formation at the University of Maryland Medical Center between 1999 and 2009. (Authors were previously affiliated with the University of Maryland.) Nontraumatic dissections
Table 1: Modified carotid artery injury grading scale

\begin{tabular}{ll}
\hline Grade & \multicolumn{1}{c}{ Description } \\
\hline 1 & Irregularity of the vessel wall or dissection/intramural \\
& hematoma with $<25 \%$ luminal stenosis \\
Intraluminal thrombus, raised intimal flap; dissection/ \\
intramural hematoma with $\geq 25 \%$ luminal narrowing \\
or hemodynamically insignificant AVF \\
a) $<70 \%$ stenosis \\
\end{tabular}

Note:-AVF indicates arteriovenous fistula.

were excluded. Fifty-three endovascular interventions were performed in 47 patients.

\section{Evaluation}

Initial evaluation for TCAI was performed with contrast-enhanced CT or conventional angiography. Injuries were evaluated with regard to patient age, sex, injury grade, injury location, complications, initial treatment results, and outcomes.

A modified Biffl classification was created and used to grade injuries. ${ }^{18}$ In our modification (Table 1), we included both blunt and penetrating injuries. Additionally, grade 2 and 3 injuries were further subdivided into $2 \mathrm{a}$ or $3 \mathrm{a}$ (non-flow-limiting when $<70 \%$ luminal narrowing was present) and $2 \mathrm{~b}$ or $3 \mathrm{~b}$ (flow-limiting when $>70 \%$ luminal narrowing was present). The degree of luminal narrowing was determined at the time of angiography by experienced neurointerventionalists on 2D cerebral angiographic images. The location of the vascular injury was divided into proximal ICA ( $>3 \mathrm{~cm}$ below the skull base) and distal ICA $(<3 \mathrm{~cm}$ from the skull base).

The criteria used for patients who underwent endovascular stent treatment included grade $2 \mathrm{~b}$ and $3 \mathrm{~b}$ injuries, progressive pseudoaneurysm enlargement, or marked intimal irregularity associated with grade 2 a injury.

\section{Procedure}

All endovascular procedures were performed in the course of routine clinical practice by experienced neurointerventional radiologists. Written informed consent was obtained for all patients, and either moderate conscious sedation or general endotracheal anesthesia was administered.

All patients received either an emergent loading bolus of 300 or $600 \mathrm{mg}$ of clopidogrel with or without $650 \mathrm{mg}$ of acetylsalicylic acid or were pretreated for 5 days with ASA, $325 \mathrm{mg}$, and clopidogrel, $75 \mathrm{mg}$. Transfemoral arterial access was used in all cases. Self-expanding stents and/or detachable platinum coils were deployed under fluoroscopic, angiographic, and roadmap guidance. Dual antiplatelet therapy (ASA, $325 \mathrm{mg}$, and clopidogrel, $75 \mathrm{mg}$ daily) was administered for a minimum of 12 weeks following stent and/or coil placement.

\section{Outcome and Follow-Up}

Initial treatment results and outcomes were classified into 4 categories: complete restoration of normal vessel lumen diameter (Fig $2)$; clinically acceptable results with mild residual pseudoaneu- 

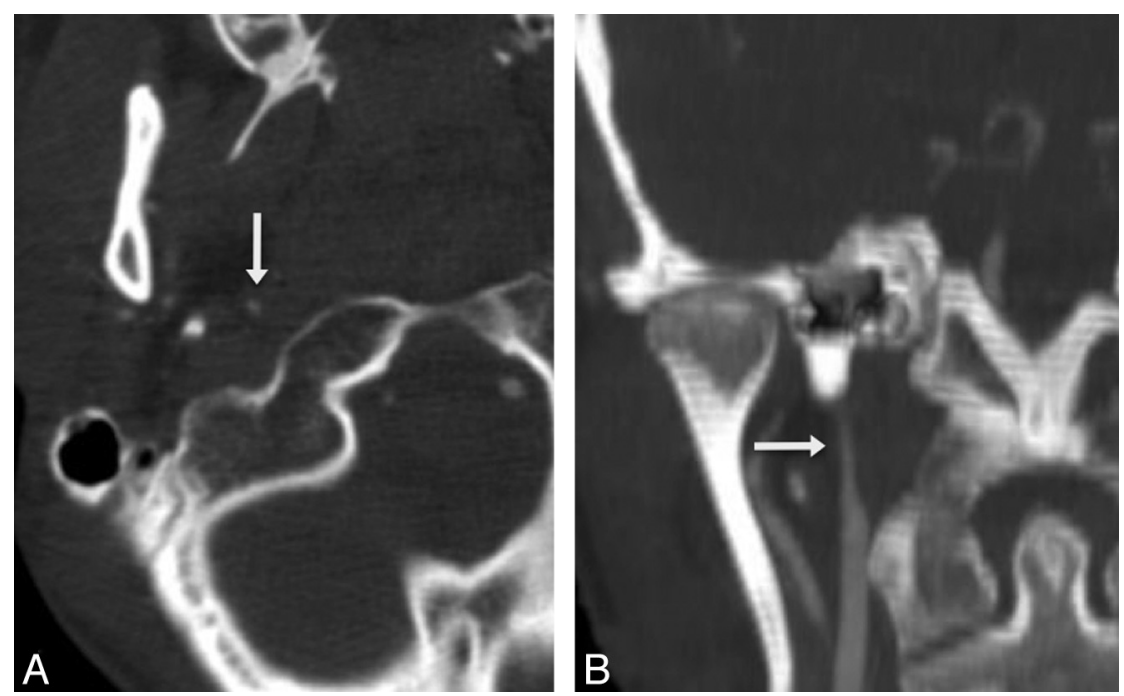

\section{Treatment}

Forty-four injuries were initially treated with stents alone, 4 initially required both stent and coil treatment, and 2 were treated with coils alone. Two patients initially treated with stents and 1 patient initially treated with stents and coils required stent and/or coil retreatment. Of the injuries treated with stent placement, 31 $(64.6 \%)$ were treated with a single stent and $17(35.4 \%)$ required multiple stents. Initial treatment resulted in complete restoration of normal vessel lumen diameter in 25 treated vessels (50\%) and good-toacceptable restoration in $25(50 \%)$ vessels. No patients were left with $>30 \%$ residual narrowing after initial treatment. Most grade 3 injuries demonstrated minimal residual pseudoaneurysm filling with acceptable restoration of demonstrate severe luminal narrowing (arrows) secondary to intramural hematoma.

rysm filling or luminal narrowing $<30 \%$; residual or recurrent narrowing $>30 \%$; and an aborted procedure or inability to deploy a stent or coils. Follow-up examinations were predominantly performed with CTA, MRA, and conventional catheter angiograms. Duplex sonography was not used due to the predominance of distal injuries within our study group.

\section{RESULTS}

\section{Patient Characteristics}

We identified 47 patients (32 men, 15 women) who underwent endovascular treatment for TCAI. Six patients had bilateral injures; thus, 53 injuries were identified. Of these 6 patients, 3 underwent bilateral endovascular treatment for a total of 50 endovascular treatments. The remaining 3 patients with bilateral injuries underwent unilateral endovascular treatment and were followed with sequential imaging. Three patients required repeat treatment, resulting in 53 endovascular interventions.

The average age at the time of treatment was 34 years and ranged from 17 to 71 years. The etiology of injury included 47 $(88.7 \%)$ blunt injuries and $6(11.3 \%)$ penetrating injuries. The number of treated vessels within each grade was as follows: grade $2 \mathrm{a}(n=5)$, grade $2 \mathrm{~b}(n=2)$, grade $3 \mathrm{a}(n=27)$, and grade $3 \mathrm{~b}(n=$ 16 ). A single grade $2 \mathrm{a}$ and 2 grade $3 \mathrm{a}$ injuries were left untreated and were stable on multiple follow-up examinations. Fifteen $(28.3 \%)$ injuries were in the proximal internal carotid artery, and $38(71.7 \%)$ injuries were in the distal cervical internal carotid artery.

Twenty-one patients initially treated with medical management required endovascular treatment due to progressive luminal narrowing or pseudoaneurysm development/enlargement. Eighteen $(87.5 \%)$ of these injuries were initially classified as grade $3 a$ injures, and $3(14.3 \%)$ were initially grade 2 a injuries. Injury progression necessitating treatment was identified, on average, within 5 weeks of the initial injury and at a maximum of 10 weeks following the initial injury. These results are summarized in Table 2. lumen caliber on immediate posttreatment angiograms.

Stent placement was aborted in a single patient with grade $3 \mathrm{a}$ injury due to underlying fibromuscular dysplasia. The patient initially presented with bilateral grade 2 a carotid injuries; however, follow-up CTA at 1 month demonstrated development of a unilateral pseudoaneurysm, and unilateral endovascular stent placement was successfully performed. Catheter angiography at 1 year demonstrated patency of the carotid stent and an unchanged dissection flap of the contralateral carotid artery with interval development of a very small pseudoaneurysm. Proximal irregularity of the vessel was consistent with fibromuscular dysplasia. Due to the overall stability of the lesion at 1 year and the presence of underlying dysplastic changes, stent placement was aborted to mitigate the risk of worsening dissection. Follow-up examination at 3 years demonstrated a stable appearance of the bilateral internal carotid arteries.

The 2 patients in whom endovascular coils were used without placement of an endovascular stent both suffered from blunt traumatic injuries. The patients were 23 and 24 years of age and the use of endovascular stents was avoided due to their age and to mitigate the need for prolonged antithrombotic therapy. The first patient presented with a grade 2 a injury, and a follow-up CT performed 2 days after the initial injury demonstrated development of a small distal cervical carotid pseudoaneurysm without flowlimiting stenosis (grade 3a). A conventional angiogram performed 2 weeks later demonstrated significant interval enlargement of the pseudoaneurysm as well as $80 \%$ narrowing of the internal carotid artery (grade $3 \mathrm{~b}$ ) (Fig $3 A,-B$ ). Nine platinum coils were placed into the pseudoaneurysm sac, and only minimal residual filling was seen on the postprocedural angiogram on the day of treatment (Fig 3C). Follow-up MRA at 6 weeks demonstrated improvement in vessel caliber and no pseudoaneurysm filling (Fig $3 D$ ). The second patient presented with a grade $3 \mathrm{a}$ injury (Fig $4 A$ ); multiple follow-up examinations for up to 3 months showed progressive pseudoaneurysm enlargement (Fig $4 B-D)$. Endovascular treatment with balloon-assisted placement of 9 platinum coils was performed, and only minimal residual 

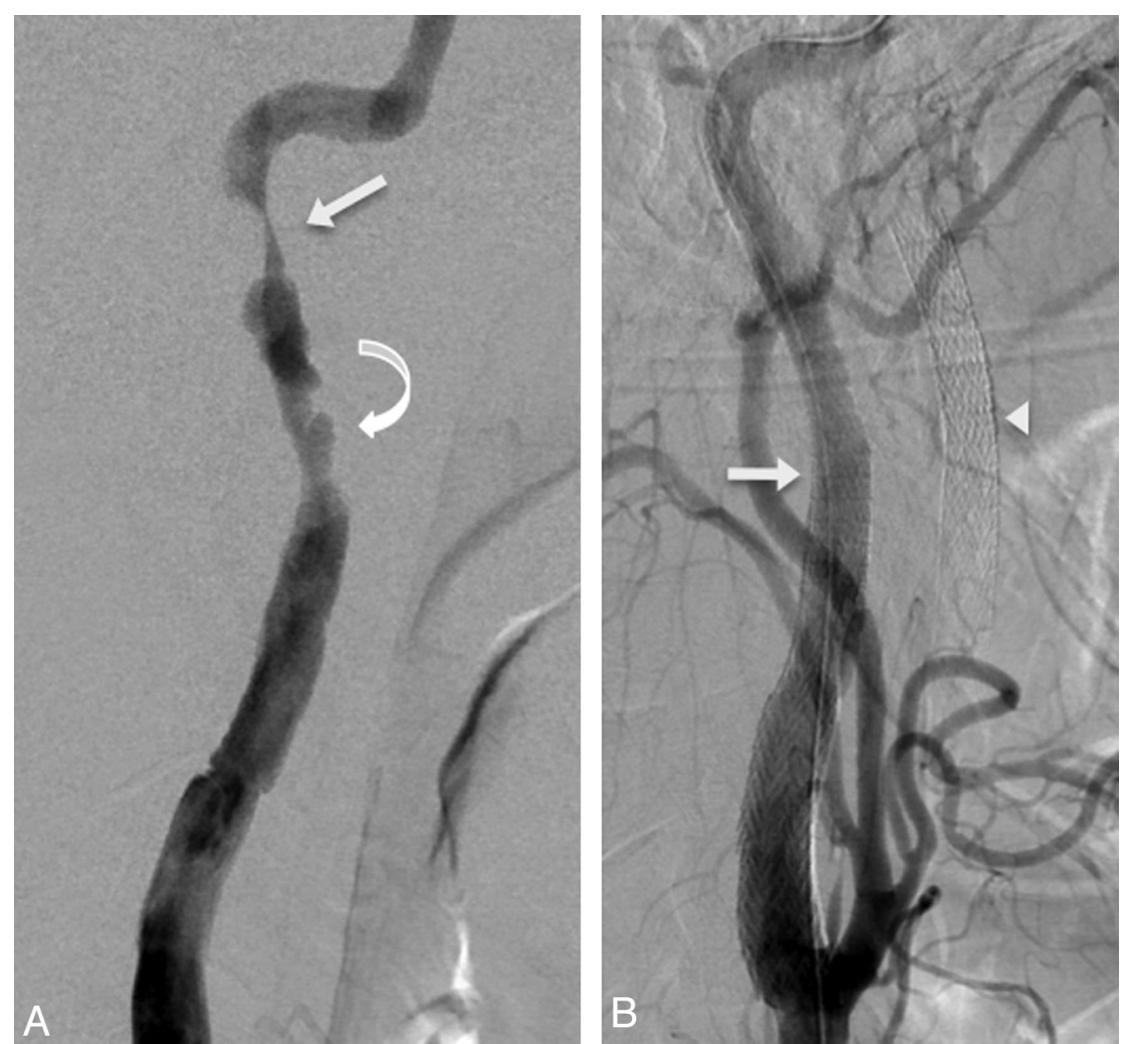

FIG 2. Stent repair, grade $3 \mathrm{~b}$ left internal carotid injury in a 43-year-old patient with bilateral carotid artery injuries following a motor vehicle collision. Lateral DSA image $(A)$ demonstrates a lengthy abnormal segment with high-grade stenosis (arrow) and multiple pseudoaneurysms (curved arrow). Lateral DSA image (B) following endovascular repair and excellent restoration of the carotid lumen with $5 \times 40$ and $6-8 \times 40 \mathrm{~mm}$ stents (arrow). Stents are also visible within the unopacified right internal carotid artery (arrowhead).

filling was seen on the postprocedural angiogram (Fig 4E). Follow-up MRA at 5.5 months after treatment demonstrated no residual pseudoaneurysm filling (Fig $4 F$ ). Neither patient had any procedural or postprocedural complication. These results are summarized in Table 3.
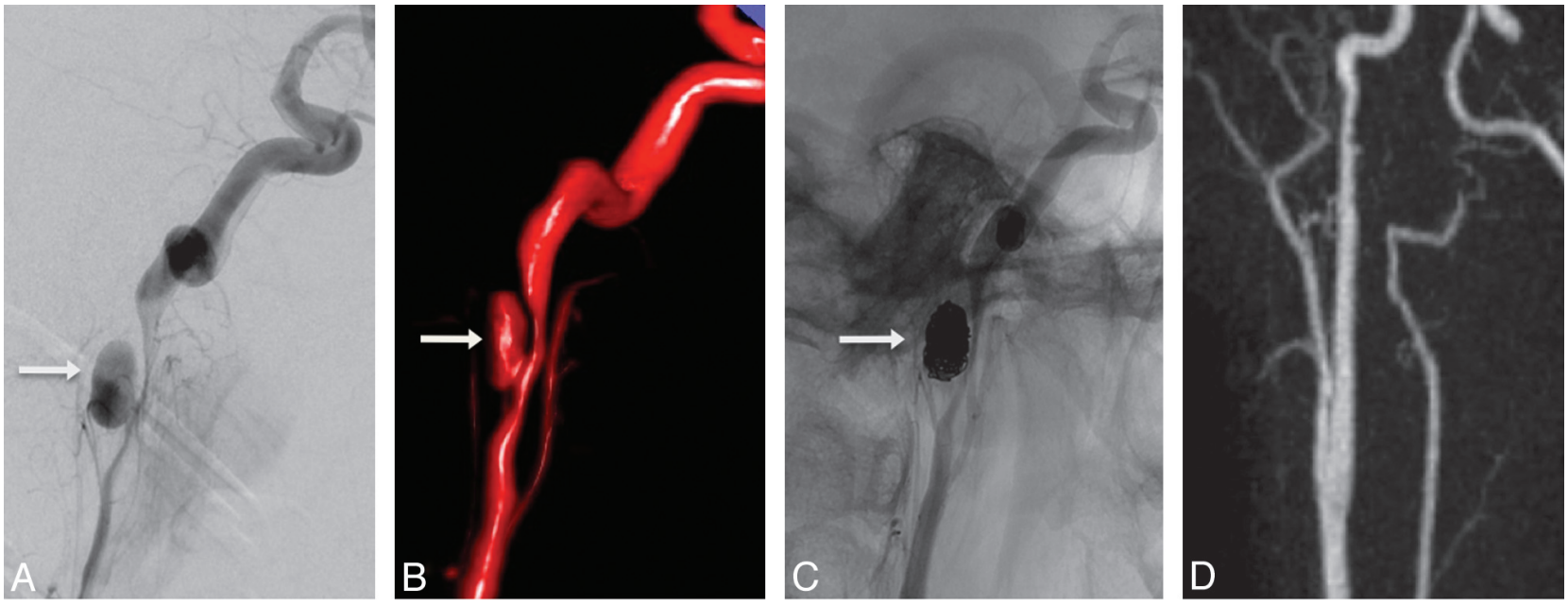

FIG 3. Endovascular coil occlusion of a carotid artery pseudoaneurysm. Grade $3 b$ right internal carotid injury in a 24-year-old patient following a motor vehicle collision. Lateral DSA $(A)$ and 3D surface-rendered image $(B)$ demonstrate a 1-cm pseudoaneurysm (arrow) with $80 \%$ luminal narrowing of the right internal carotid artery. Posttreatment angiogram (C) following balloon-assisted coiling with placement of 9 endovascular coils demonstrates complete occlusion of the pseudoaneurysm. Postcontrast MRA $(D) 3$ weeks after treatment demonstrates durable occlusion of the pseudoaneurysm and substantial improvement in the right internal carotid artery caliber without stent placement. 

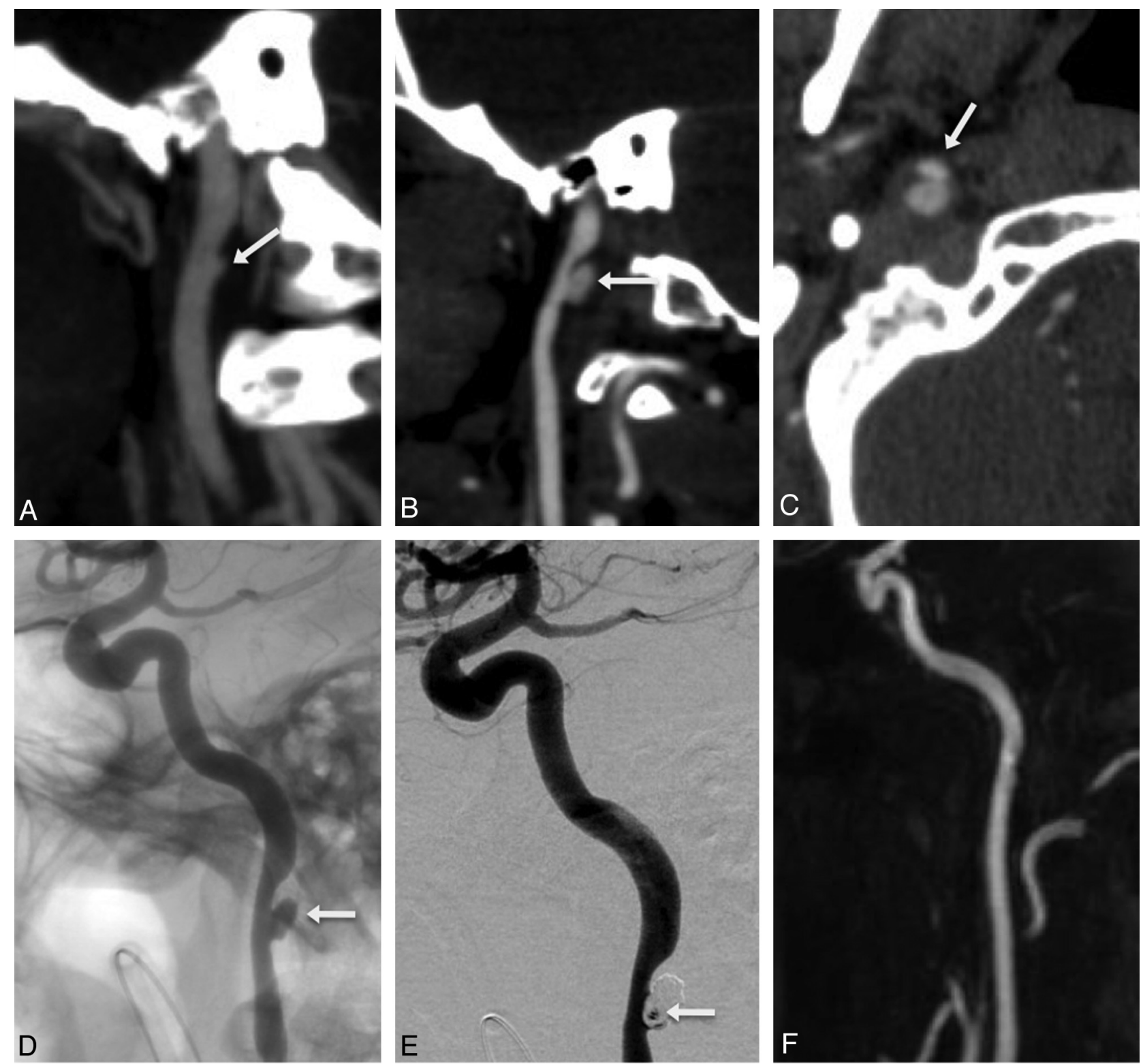

FIG 4. Endovascular coil occlusion of an enlarging carotid artery pseudoaneurysm. Sagittal CTA reconstruction (A) of a 23-year-old man status post motorcycle collision demonstrates a small blister pseudoaneurysm of the distal right internal carotid artery. Follow-up sagittal (B) and axial (C) CTA and lateral angiographic (D) images show interval pseudoaneurysm enlargement (arrows) without flow-limiting stenosis. Posttreatment DSA (E) following placement of 9 detachable coils demonstrates minimal residual filling of the aneurysm sac (arrow). Postcontrast MRA (F) at 5.5 months demonstrates improvement in the vascular caliber and no residual pseudoaneurysm filling.

tervention. Resolution of spasm following immediate intra-arterial therapy and patency of the stent were documented by angiography at the termination of the case. However, follow-up CTA on postprocedural day 8 demonstrated complete stent occlusion. Collateral intracranial flow through the circle of Willis was present, and follow-up MR imaging on postprocedure day 14 demonstrated no evidence of infarction. The patient remains clinically asymptomatic from the occlusion.

A single patient $(2.1 \%)$ initially treated for a grade $2 \mathrm{~b}$ dissection of the proximal right internal carotid artery demonstrated a new pseudoaneurysm and some luminal narrowing on follow-up CTA performed 1 week following stent placement (Fig 5A, - B). A catheter angiogram confirmed these findings, and luminal restoration with near-complete occlusion of the pseudoaneurysm sac was achieved with coil embolization and placement of an additional stent (Fig 5C).

Two of the treated patients $(4.3 \%)$ required repeat intervention for unhealed initial injuries. The first patient underwent stent placement for a lengthy grade 3 a dissection and pseudoaneurysm with 50\% narrowing of the mid-to-distal left internal carotid artery secondary to a motor vehicle collision. Follow-up angiography 1 week later demonstrated persistent filling of the pseudoaneurysm sac. Complete occlusion was achieved with coil embolization and placement of an additional stent. The second patient had a focal grade $2 \mathrm{a}$ dissection of the mid-to-distal left internal carotid artery and was initially treated conservatively. Follow-up CTA at 2 months demonstrated progression of the dissection with formation of a new 3-mm pseudoaneurysm, and 

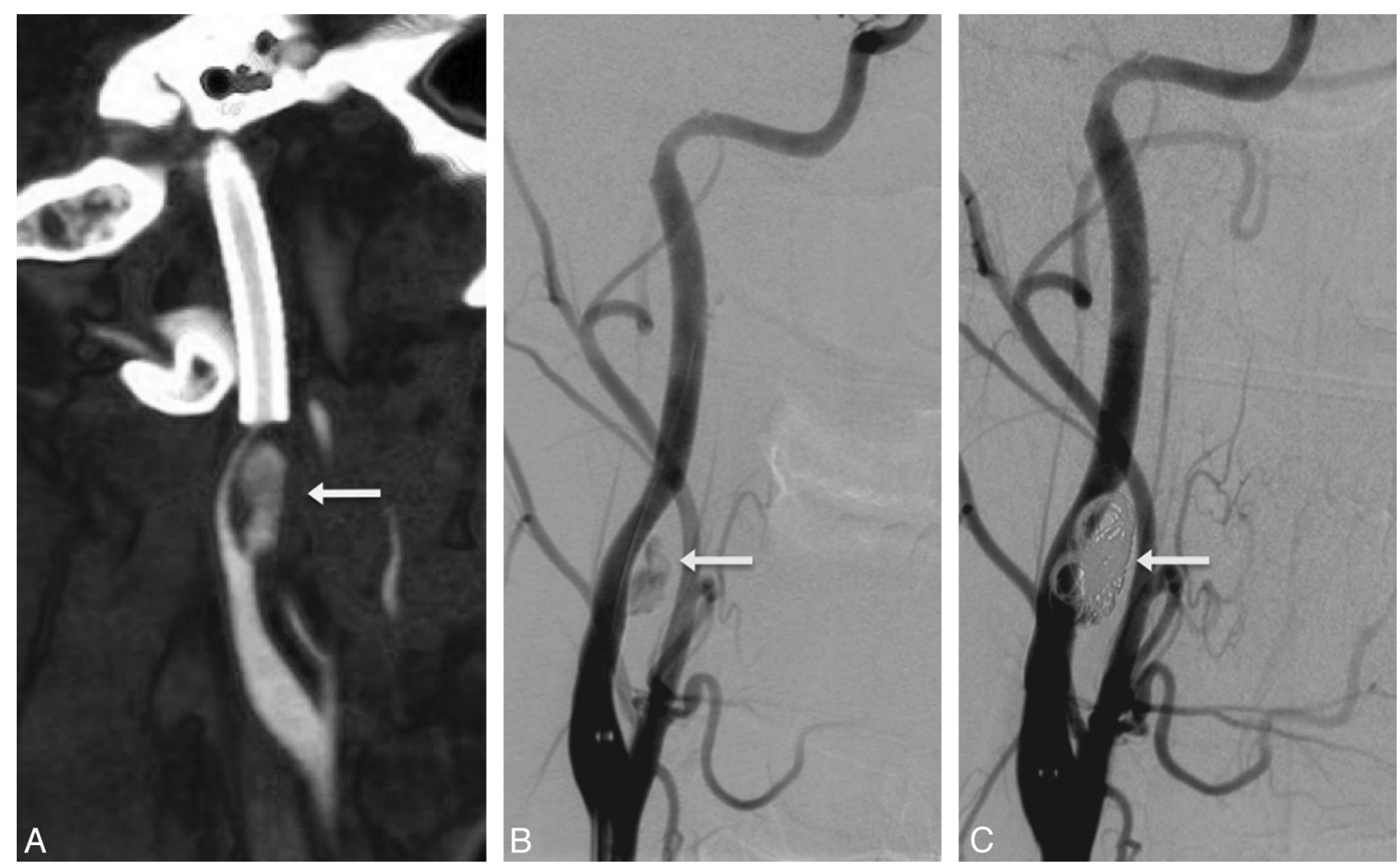

FIG 5. Retreatment (stent-coiling) of a new carotid pseudoaneurysm 1 week after initial stent placement. Lateral CTA reconstruction ( $A$ ) of the right internal carotid artery in a 21-year-old patient treated 1 week earlier for grade $2 \mathrm{~b}$ injury of the right internal carotid artery following a motor vehicle collision. Pseudoaneurysm (arrow) and high-grade stenosis developed proximal to the initial stent. Posttreatment lateral DSA (B) after placement of an additional stent demonstrates persistent filling of the pseudoaneurysm (arrow). DSA after placement of a stent and 4 detachable coils $(C)$ demonstrates complete occlusion of the pseudoaneurysm.

endovascular treatment with a stent and 4 platinum coils was performed. A follow-up catheter angiogram at 5 weeks demonstrated in-stent intimal hyperplasia and $40 \%$ luminal narrowing, as well as an enlarging pseudoaneurysm. Complete occlusion of the sac was achieved with placement of a second stent and 6 additional platinum coils. A follow-up catheter angiogram at 1.5 years demonstrated unchanged intimal hyperplasia with no residual dissection or pseudoaneurysm filling.

No groin-related complications were identified. There were no deaths or permanent morbidity relating to endovascular carotid artery repair.

\section{DISCUSSION}

Although risk factors and screening recommendations have been suggested, ${ }^{4}$ the appropriate choice of treatment for TCAI remains debated. Antithrombotic and antiplatelet agents are advocated in patients without contraindications and have been shown to decrease the incidence of neurologic sequelae and mortality related to carotid injury when compared with no treatment. ${ }^{14,19,20}$ In patients that underwent surgical treatment, surgical repair resulted in a lower mortality rate $(7.8 \%)$ when compared to surgical ligation $(50 \%) .{ }^{21}$ However, patients with severe neurologic deficits have not demonstrated a significant improvement in outcome with either surgical treatment or anticoagulation therapy. ${ }^{22}$

In patients with only cervical vascular injury, treatment typically consists of a choice between antithrombotic therapy and endovascular repair. However, endovascular repair with stents also requires pre- and posttreatment antiplatelet therapy to avoid embolic complications or stent occlusion. This often results in a frustrating clinical conundrum because many patients are referred for endovascular repair specifically due to contraindications to antithrombotic therapy such as severe intracranial injury, multisystem trauma, or penetrating injuries. Unless faced with an immediately life-threatening injury, our practice has been to avoid placement of stents in patients who have not or cannot receive appropriate pre- and postprocedural antiplatelet therapy.

Biffl et $\mathrm{al}^{23}$ in 2002 advocated a 7- to 10-day angiographic follow-up on the basis of their observation that most grade 1 or 2 injuries demonstrated either complete healing or progression within that time period. In our practice, we typically recommend CTA follow-up within 3-5 days for patients treated with antiplatelet medications alone. Additional follow-up CTA was acquired 1 week later and at increasing intervals until stability of the injury was documented or a decision to intervene was made.

Only limited prospective data are available regarding the safety and efficacy of carotid artery stent placement in the setting of trauma, and most published results are from small case review series. A single prospective study by Cothren et $\mathrm{al}^{17}$ showed discouraging results with a high rate of stent occlusion; however, we believe that the lack of antiplatelet therapy in patients with complications may have played a role in those poor outcomes.

Published retrospective studies, on the other hand, have shown encouraging results. A series of 6 patients treated with endo- 
Table 2: Demographics and characterization of carotid artery injuries

\begin{tabular}{lc}
\hline \multicolumn{1}{c}{ Demographics } & Data \\
\hline Total patients & 47 \\
Total cervical internal carotid artery injuries & 53 \\
Total treated cervical internal carotid artery injuries & 50 \\
Average age (yr) & 34 \\
Age range (yr) & $17-71$ \\
Sex & \\
$\quad$ Male & $32(68.1 \%)$ \\
Female & $15(31.9 \%)$ \\
Etiology of injury & \\
Blunt trauma & $47(88.7 \%)$ \\
Penetrating trauma & $6(11.3 \%)$ \\
Side of injury & \\
Right only & $24(51.1 \%)$ \\
Left only & $17(37.2 \%)$ \\
Bilateral & $6(12.8 \%)$ \\
Location of injury & \\
Proximal & $15(28.3 \%)$ \\
Distal & $38(71.7 \%)$ \\
Grade of injury (treated) & \\
2a & $5(10.0 \%)$ \\
2b & $2(4.0 \%)$ \\
3a & $27(54.0 \%)$ \\
3b & $16(32.0 \%)$ \\
\hline
\end{tabular}

vascular stent placement for enlarging pseudoaneurysms reported by Duke et $\mathrm{al}^{24}$ in 1997 showed complete healing in all patients, suggesting that endovascular stents were a safe and effective alternative to surgery. A more recent and larger series reported 29 procedures with only 2 long-term complications; once again, both patients who had complications had not received antiplatelet medications. ${ }^{16}$ In a small study of 7 patients with a 3.5-year follow-up, Liu et $\mathrm{al}^{25}$ reported no postprocedural complications and a single asymptomatic occlusion at 3 months. A case review series of 14 patients by Coldwell et $\mathrm{al}^{26}$ in 2000 demonstrated no procedural complications and complete healing of all treated vessels at 4-month follow-up.

Our experience with cervical vascular injury at a large trauma center has been substantial. To our knowledge, this report represents the largest published case series review of endovascular treatment for TCAI. In our study population, blunt injuries were 9 times more frequent than penetrating injuries, and men outnumbered women by $>2$ to 1 , reaffirming that men are at higher risk than women for traumatic carotid injury. There were transient postprocedural complications in 3 of 50 procedures $(6.0 \%)$. A single stent placement procedure (2.0\%) resulted in delayed asymptomatic vessel occlusion, and follow-up imaging at 2 months and clinical follow-up at 1 year demonstrated no evidence of cerebral infarction. One patient (2\%) developed a new pseudoaneurysm and was successfully treated endovascularly. Although a small amount of residual filling of the pseudoaneurysm sac was frequently seen after initial treatment, follow-up studies showed complete occlusion of the sac in nearly all of our patients. Only 2 of the 47 treated patients ( $4.3 \%$ ) required additional endovascular treatment for residual pseudoaneurysm filling, thus demonstrating the durability of successful initial endovascular repair. Overall, various grades of injury were treated successfully, with minimal complications and no mortality related to this repair.

As with most retrospective reviews, there are limitations to our study. Specifically, there was significant variability in the time
Table 3: Treatment and follow-up of dissections and dissecting aneurysms

\begin{tabular}{|c|c|}
\hline Treatment and Follow-Up & Data \\
\hline \multicolumn{2}{|l|}{ Treatment method (initial) } \\
\hline Stent only & $44(88.0 \%)$ \\
\hline Coils only & $2(4.0 \%)$ \\
\hline Combination stent and coils & $4(8.0 \%)$ \\
\hline \multicolumn{2}{|l|}{ Stent therapy } \\
\hline Single & $31(64.6 \%)$ \\
\hline Multiple & $17(35.4 \%)$ \\
\hline \multicolumn{2}{|l|}{ Result (immediate) } \\
\hline $\begin{array}{l}\text { Complete luminal restoration/pseudoaneurysm } \\
\text { occlusion }\end{array}$ & $25(50.0 \%)$ \\
\hline $\begin{array}{l}\text { Acceptable luminal restoration/minimal } \\
\text { pseudoaneurysm filling }\end{array}$ & $25(50.0 \%)$ \\
\hline Persistent high-grade stenosis (<30\%) & 0 \\
\hline \multicolumn{2}{|l|}{ Complications } \\
\hline Stent occlusion & $1(2.0 \%)$ \\
\hline Stent-related pseudoaneurysm & $1(2.0 \%)$ \\
\hline Transient weakness & $2(4.0 \%)$ \\
\hline Transient visual loss & $1(2.0 \%)$ \\
\hline \multicolumn{2}{|l|}{ Follow-up imaging (No. of exams) } \\
\hline CTA & 64 \\
\hline MRA & 17 \\
\hline Conventional angiogram & 23 \\
\hline \multicolumn{2}{|l|}{ Follow-up imaging (No. of patients) } \\
\hline CTA or MRA & $39(83.0 \%)$ \\
\hline CTA and MRA & $9(9.0 \%)$ \\
\hline Conventional angiogram & $15(31.9 \%)$ \\
\hline No follow-up & $7(14.9 \%)$ \\
\hline \multicolumn{2}{|l|}{ Retreatment } \\
\hline Injuries requiring repeat treatment & $3(6.0 \%)$ \\
\hline Average time to retreatment (wk) & 3 \\
\hline $\begin{array}{l}\text { Initially untreated injuries requiring treatment } \\
\text { on follow-up }\end{array}$ & $21(42.0 \%)$ \\
\hline Initial Grade 2a & $3(14.3 \%)$ \\
\hline Initial Grade 3a & $18(85.7 \%)$ \\
\hline $\begin{array}{l}\text { Average time to treatment for initially untreated } \\
\text { injuries (wk) }\end{array}$ & 5 \\
\hline
\end{tabular}

interval at which follow-up examinations were obtained, and no imaging follow-up was performed in 7 patients. In addition, there was significant variability in the technique and imaging protocols used to follow either treated or untreated injuries.

The 2 identified complications directly related to endovascular therapy occurred within the first 2 weeks after treatment. In patients treated with endovascular stents, only 2 patients had progressive pseudoaneurysm enlargement necessitating intervention. Both of these patients were identified within 5 weeks of the initial treatment and were successfully treated with endovascular pseudoaneurysm coil occlusion. In patients with long-term follow-up, there were no significant late-occurring adverse effects related to treatment. Taken together, these findings indicate a need for close surveillance in the perioperative period and extending to 60 days. Most of our stented patients are now maintained on dual oral antiplatelet agents (ASA, $325 \mathrm{mg}$, and clopidogrel bisulfate, $75 \mathrm{mg}$ daily) for 12 weeks, at which time a follow-up imaging study is performed. If follow-up imaging demonstrates a stable or improving appearance, patients are then maintained on ASA, $81 \mathrm{mg}$, daily for life. The mean age of 34 years in our study population is distinctly different from the population typically undergoing stent placement for atherosclerotic carotid stenosis, and concerns about life-long antiplatelet therapy should guide 
treatment decisions in individual cases. Potential morbidity related to life-long oral antiplatelet therapy and the risk of noncompliance for many years are concerning. Taking this into consideration, 2 young patients (23 and 24 years of age) with grade 3 injuries were treated with coil placement alone to avoid the need for life-long antiplatelet therapy. Both patients showed no residual pseudoaneurysm and had no postprocedural complications. Furthermore, follow-up imaging demonstrated significant healing of the carotid artery, with improvement in vessel caliber in both cases. We suspect that this improvement in caliber is due to diminished perivascular inflammation and related mass effect on the parent artery following effective endovascular treatment.

Although the optimal treatment for extracranial carotid artery dissection with or without pseudoaneurysm remains controversial, our study adds to the previously published data demonstrating that endovascular therapy is a safe and effective method of restoring luminal caliber in traumatic carotid artery injuries. Continued investigation is required to better stratify patients into groups benefiting most from medical management and those in whom endovascular repair is necessary. With the addition of our modification of the Biffl scale to account for high-grade stenosis, our study demonstrated a subset of patients in whom either intervention or close interval follow-up may be required. Specifically, of the 21 patients initially treated with medical management who required endovascular treatment due to injury progression, $18(85.7 \%)$ had grade $3 a$ injures and $3(14.3 \%)$ had grade $2 \mathrm{a}$ injuries. On average, progression of injury grade necessitating treatment was identified within 5 weeks of the initial injury. We, therefore, suggest that imaging follow-up of all carotid injuries, especially those deemed to be grades $3 \mathrm{a}$ and $2 \mathrm{a}$ injuries, should be performed within the critical period of the first 45 days after injury.

\section{CONCLUSIONS}

We reviewed 50 extracranial internal carotid injuries that were successfully treated with endovascular therapy consisting of stent placement or stent-assisted coil placement. Endovascular therapy is a safe and effective option for rapidly restoring luminal caliber and eliminating flow within pseudoaneurysms related to traumatic injuries of the cervical internal carotid artery. Both flowlimiting and non-flow-limiting dissections and pseudoaneurysm formation demonstrate durable healing after treatment. Imaging follow-up of all cervicocerebral vascular injuries is especially important within the first 45 days, a critical interval during which most lesions demonstrate healing or progression. Noninvasive imaging such as CTA, MR imaging, and MRA was useful for initial diagnosis and follow-up. Complications were rare and did not result in permanent morbidity or mortality. We attribute the low rate of complications to our routine use of oral antiplatelet medications before and following endovascular treatment.

\section{REFERENCES}

1. Stein DM, Boswell S, Sliker CW, et al. Blunt cerebrovascular injuries: does treatment always matter? J Trauma 2009;66:132-43, discussion 143-44

2. Ducrocq X, Lacour JC, Debouverie M, et al. Cerebral ischemic acci- dents in young subjects: a prospective study of 296 patients aged 16 to 45 years [in French]. Rev Neurol (Paris) 1999;155:575-82

3. Berne JD, Cook A, Rowe SA, et al. A multivariate logistic regression analysis of risk factors for blunt cerebrovascular injury. J Vasc Surg 2010;51:57-64

4. Eastman AL, Chason DP, Perez CL, et al. Computed tomographic angiography for the diagnosis of blunt cervical vascular injury: is it ready for primetime? J Trauma 2006;60:925-29

5. Cogbill TH, Moore EE, Meissner M, et al. The spectrum of blunt injury to the carotid artery: a multicenter perspective. $J$ Trauma 1994;37:473-79

6. Berne JD, Norwood SH, McAuley CE, et al. Helical computed tomographic angiography: an excellent screening test for blunt cerebrovascular injury. J Trauma 2004;57:11-17, discussion 17-19

7. Miller PR, Fabian TC, Croce MA, et al. Prospective screening for blunt cerebrovascular injuries: analysis of diagnostic modalities and outcomes. Ann Surg 2002;236:386-93, discussion 393-95

8. Mutze S, Rademacher G, Matthes G, et al. Blunt cerebrovascular injury in patients with blunt multiple trauma: diagnostic accuracy of duplex Doppler US and early CT angiography. Radiology 2005; 237:884-92

9. Prall JA. Incidence of unsuspected blunt carotid artery injury. $\mathrm{Neu}$ rosurgery 1998;42:495-98, discussion 498-99

10. Biffl WL. The devastating potential of blunt vertebral arterial injuries. Ann Surg 2000;231:672-81

11. Schievink WI. Spontaneous dissection of the carotid and vertebral arteries. N Engl J Med 2001;344:898-906

12. Schievink WI, Mokri B, Whisnant JP. Internal carotid artery dissection in a community: Rochester, Minnesota, 1987-1992. Stroke 1993;24:1678-80

13. Biffl WL, Moore EE, Offner PJ, et al. Optimizing screening for blunt cerebrovascular injuries. Am J Surg 1999;178:517-22

14. Cothren CC, Moore EE, Biffl WL, et al. Anticoagulation is the gold standard therapy for blunt carotid injuries to reduce stroke rate. Arch Surg 2004;139:540-45, discussion 545-46

15. Wahl WL, Brandt MM, Thompson BG, et al. Antiplatelet therapy: an alternative to heparin for blunt carotid injury. J Trauma 2002;52: 896-901

16. Kadkhodayan Y, Jeck DT, Moran CJ, et al. Angioplasty and stenting in carotid dissection with or without associated pseudoaneurysm. AJNR Am J Neuroradiol 2005;26:2328-35

17. Cothren CC, Moore EE, Ray CE, et al. Carotid artery stents for blunt cerebrovascular injury: risks exceed benefits. Arch Surg 2005;140: 480-85, discussion 485-86

18. Biffl WL, Moore EE, Offner PJ, et al., Blunt carotid arterial injuries: implications of a new grading scale. J Trauma 1999;47:845-53

19. Fabian RC, Patton JH Jr, Croce MA, et al. Blunt carotid injury: importance of early diagnosis and anticoagulant therapy. Ann Surg 1996;223:513-22, discussion 522-25

20. Miller PR, Fabian TC, Bee TK, et al. Blunt cerebrovascular injuries: diagnosis and treatment. J Trauma 2001;51:279-85, discussion 285-86

21. Karlin RM, Marks C. Extracranial carotid artery injury: current surgical management. Am J Surg 1983;146;225-27

22. Richardson JD, Simpson C, Miller FB. Management of carotid artery trauma. Surgery 1988;104:673-80

23. Biffl WL, Ray CE, Moore EE, et al. Treatment-related outcomes from blunt cerebrovascular injuries: importance of routine follow-up angiography. Ann Surg 2002;235:699-06, discussion 706-07

24. Duke BJ, Ryu RK, Coldwell DM, et al. Treatment of blunt injury to the carotid artery by using endovascular stents: an early experience. J Neurosurg 1997;87:825-29

25. Liu AY, Paulsen RD, Marcellus ML, et al. Long-term outcomes after carotid stent placement treatment of carotid artery dissection. $\mathrm{Neu}$ rosurgery 1999;45:1368-73, discussion 1373-44

26. Coldwell DM, Novak Z, Ryu RK, et al. Treatment of posttraumatic internal carotid arterial pseudoaneurysms with endovascular stents. J Trauma 2000;48:470-72 